\title{
INVESTIGATION ON THE EFFECT OF MONOGLYCERIDE CONCENTRATION ON PALM OIL-BASED ALKYD RESIN PREPARATION
}

\author{
RIDZUAN RAMLI*; HUEI RUEY ONG**; CHI SHEIN HONG ; MD. MAKSUDUR RAHMAN KHAN ${ }^{\ddagger \neq}$; \\ ROSLI MOHD YUNUS*; ROHAYA MOHAMED HALIM*; ASTIMAR ABDUL AZIZ*; ZAWAWI IBRAHIM* \\ and MUHAMMAD REMANUL ISLAM ${ }^{\ddagger \ddagger}$
}

\begin{abstract}
Alcoholysis and esterification are useful methods to prepare polyalkyds from vegetable oils. Reaction parameters and different concentration of raw materials have showed a significant role on the properties of the formulated polyalkyds. The formation of monoglyceride (MG) and diglyceride (DG) during the synthesis and their ratio may have a significant role on the properties of the polyalkyds. The goal of the present article was to investigate the effect of $M G$ to DG ratio on alkyd resin synthesised from palm oil by alcoholysispolyesterification reactions catalysed by homo-hetero catalytic system. Different ratios of MG:DG were obtained from alcoholysis reaction by taking samples at different reaction times. The formation of the alkyd resin was confirmed by Fourier transform infrared spectroscopy (FTIR) with the presence of ester group (C-O-C). The molecular weight $\left(M_{w}\right)$ of alkyd resin was determined by gel permeation chromatography (GPC). The alkyd resin prepared at higher MG:DG ratio exhibited higher initial rate of polyesterification reaction with higher $M_{w}$. In addition, the film of alkyd resin prepared from higher MG:DG ratio presented better pencil hardness and chemical resistivity.
\end{abstract}

Keywords: alkyd resin, CuO nanoparticles, FTIR, monoglyceride, palm oil.

Received: 14 May 2020; Accepted: 10 August 2020; Published online: 7 October 2020.

\section{INTRODUCTION}

Alkyd resins are very important materials in coating industries. They have been used in varnishes,

\footnotetext{
* Malaysian Palm Oil Board,

6 Persiaran Institusi, Bandar Baru Bangi,

43000 Kajang, Selangor, Malaysia.

E-mail: ridz@mpob.gov.my

** DRB-Hicom University of Automotive Malaysia, Lot 1449, PT2204, Peramu Jaya Industrial Area, 26607 Pekan, Pahang, Malaysia.

‡ Faculty of Chemical and Natural Resources Engineering, Universiti Malaysia Pahang, Lebuhraya Tun Razak, 26300 Kuantan, Pahang, Malaysia.

\#‡ Universiti Kuala Lumpur, Malaysian Institute of Industrial, Technology (UniKL MITEC), Persiaran Sinaran IImu, Bandar Seri Alam, 81750 Johor Bahru, Johor, Malaysia.
}

composite coatings, lacquers and adhesives industries (Dolui, 2015; Meneghetti and Meneghetti, 2015). Triglyceride (TG) based vegetable oil can be used to synthesis alkyd resins. To synthesis alkyd resin in the laboratory, alcholysis is the first step, which followed by esterification (Mutar and Hassan, 2017). For the alcoholysis process, the most common polyol used is glycerol, whereas, for esterification phthalic anhydride is widely used (Ong et al., 2018). Different types of catalysts like lead(II) oxide $(\mathrm{PbO})$, potassium hydroxide $(\mathrm{KOH})$, zinc oxide $(\mathrm{ZnO})$, lithium oxide $(\mathrm{LiO})$, calcium oxide $(\mathrm{CaO})$ and sodium hydroxide $(\mathrm{NaOH})$ can be used for the reactions. The reported reaction temperature is around $240^{\circ} \mathrm{C}$. The conversion in the first step, after the alcoholysis, the reaction products usually contain 40\%-60\% monoglyceride (MG) (Rarokar et al., 2017). This MG is required to homogenise the reaction medium which is a favourable condition 
for the second step, polyesterification. MG and diglyceride (DG) reaction with polyacid can form the linear polymer. The chain length of the alkyd resin is related to $\mathrm{MG}: \mathrm{DG}$ ratio, where $\mathrm{DG}$ acts as a chain stopper.

Several numbers of researches have investigated the polyalkyds preparation from vegetable oils using the two-step process, alcoholysis and esterification (Beg et al., 2019). Optimisation of the reaction parameters and characterisation of the synthesised polyalkyds were the focus of these works. Some of the works highlighted about the drying and non-drying properties of the vegetable oils. In addition, further modification of the polyalkyds was also suggested. Based on the previous results, it was hypothesised that the esterification can be influenced by the factor of MG and DG ratio. Bearing this in mind, the current research work has been developed. Moreover, to the best of our knowledge, other researchers preferred the methanol solubility method during alcoholysis reaction, but this method does not quantify the MG concentration. Recently, our laboratory prepared MG from palm oil via copper(II) oxide - sodium hydroxide $(\mathrm{CuO}-\mathrm{NaOH})$ catalyst system and using high performance liquid chromatography (HPLC) method to quantify the MG concentration (Ong et al., 2016a). The effect of MG:DG ratio on alkyd preparation has been undermined throughout the literature. In this context, the goal of this work is to investigate the effect of MG:DG ratio on polyesterification reaction. Moreover, the pencil hardness and chemical resistivity of the alkyd films were elucidated.

\section{METHODOLOGY}

\section{Materials}

The chemicals and reagents used in this study were ethanol (99.9\%), copper (II) chloride salt $\left(\mathrm{CuCl}_{2} .2 \mathrm{H}_{2} \mathrm{O}\right)$, standard 1-oleoyl-rac-glycerol $(\geq 99 \%), \mathrm{NaOH}$, hydrazine monohydrate $(64 \%)$, glycerol, cobalt octoate, $\mathrm{KOH}, 1,2$-dioleoyl-snglycerol $(\geq 97 \%)$, methyl ethyl ketone peroxide (MEKP), styrene, and glyceryl trioleate $(\geq 99 \%)$ which were purchased from Sigma-Aldrich, Malaysia. Acetone and acetonitrile were procured from Merck (Malaysia) Sdn. Bhd. Refined palm oil was kindly supplied by Malaysian Palm Oil Board (MPOB), Malaysia. All chemicals were used in this research as they were received. Figure 1 depicts the flow diagram of methodology for this study.

\section{Preparation of $\mathrm{CuO}$ Nanoparticles}

The $\mathrm{CuO}$ nanoparticles were synthesised at ambient temperature by sol-gel method (Ong et al., 2015a; 2015b). First of all, a fixed amount of $\mathrm{CuCl}_{2} \cdot 2 \mathrm{H}_{2} \mathrm{O}$ was dissolved in $44 \mathrm{ml}$ of glycerol. After that, $6 \mathrm{ml}$ of hydrazine solution was added into the mixture dropwise. The concentration of copper was maintained from 100-300 mg litre ${ }^{-1}$. Hydrazine solution $(7.062 \mathrm{mM})$ was prepared earlier by diluting $64 \%$ of hydrazine monohydrate in ethanol.

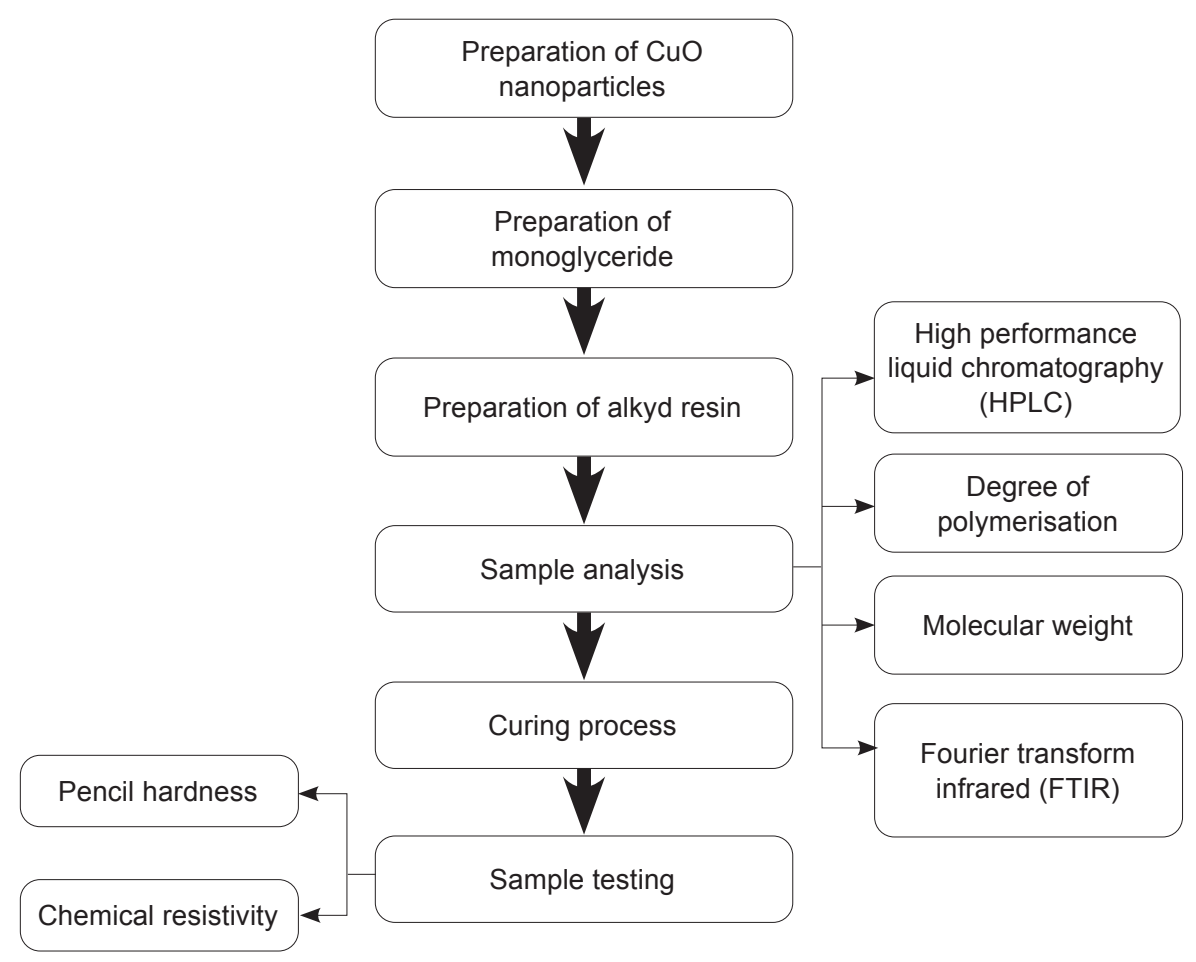

Figure 1. Flow diagram of methodology. 


\section{Preparation of Monoglyceride}

Alcoholysis process was adopted to prepare the MG from palm oil and glycerine. In the process, nearly $100 \mathrm{~g}$ of palm oil was poured in a beaker with $\mathrm{CuO}$ nano particles (22.2 g). The mixture was stirred continuously and heated at $240^{\circ} \mathrm{C}$. After that, additionally, a little amount of $\mathrm{NaOH}(0.3$ g) was added. The reaction was conducted in a continuous flow of nitrogen gas into the reaction chamber.

\section{Preparation of Alkyd Resin}

After the completion of alcoholysis reaction, the temperature of the reactor was reduced to $140^{\circ} \mathrm{C}$. After that, $38.7 \mathrm{~g}$ of phthalic anhydride was added into the reactor. Then, the temperature of the reactor was increased to $240^{\circ} \mathrm{C}$. The acid value (AV) of the products was determined by ASTM D1639-90, at different times during the propagation of the reaction. When the $\mathrm{AV}$ reached to nearly $10 \mathrm{mg} \mathrm{KOH} \mathrm{g}^{-1}$, then the reactor was cooled down. To investigate the effect of MG:DG ratio on polyesterification reaction, the alcoholysis reaction was stopped at different times (30, 40 and $60 \mathrm{~min}$ ) and the polyesterification reaction was conducted as mentioned above. The synthesis pathway of alkyd resin is presented in Figure 2.

\section{High Performance Liquid Chromatography (HPLC)}

The HPLC (Agilent 1200 Series) was used to quantify the reaction products. The machine was equipped with refractive index detector (RID) and diode-array detectors (DAD). A Zorbax C18 column with the specification of $4.6 \mathrm{~m} \times 250 \mathrm{~mm}$ $(5 \mu \mathrm{m})$ was used for the analysis. The flow rate was

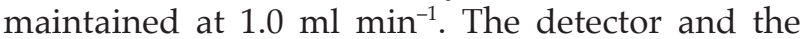
column temperature were fixed to $40^{\circ} \mathrm{C}$ and $35^{\circ} \mathrm{C}$, respectively. A mixture of acetone and acetonitrile $(65: 35, v / v)$ was used as a carrier of the sample through the column. Another composition of acetone and acetonitrile $(1: 1, \mathrm{v} / \mathrm{v})$ was used to dissolve the sample. The volume of the injection was $10 \mu \mathrm{l}$. The standards of MG, DG and TG were used to quantify the reaction products. Different concentrations at $300,800,1000,2000,5000,8000$ and 10000 ppm were used to prepare the calibration curves. At least three different runs were carried out for each analysis. A slow agitation and slightly warmed samples were used for the analysis to avoid the separation of the phases. The experimental deviations were considered less than $5 \%$ for the content of MG, DG and TG.

\section{Degree of Polymerisation Determination}

During the esterification reaction, the reaction products were collected for the AV testing. The titrimetric value was used for the determination of AV. The AV was used to calculate the extent of the reaction, $P$. The average degree of polymerisation (DP) was calculated using the value of $P$. The following equations were used for the calculation:

$$
\begin{gathered}
P=\left(C_{0}-C_{t}\right) / C_{0}--(1) \\
D P=(1-P)^{-1}--(2)
\end{gathered}
$$

First Stage: Alchoholysis Process
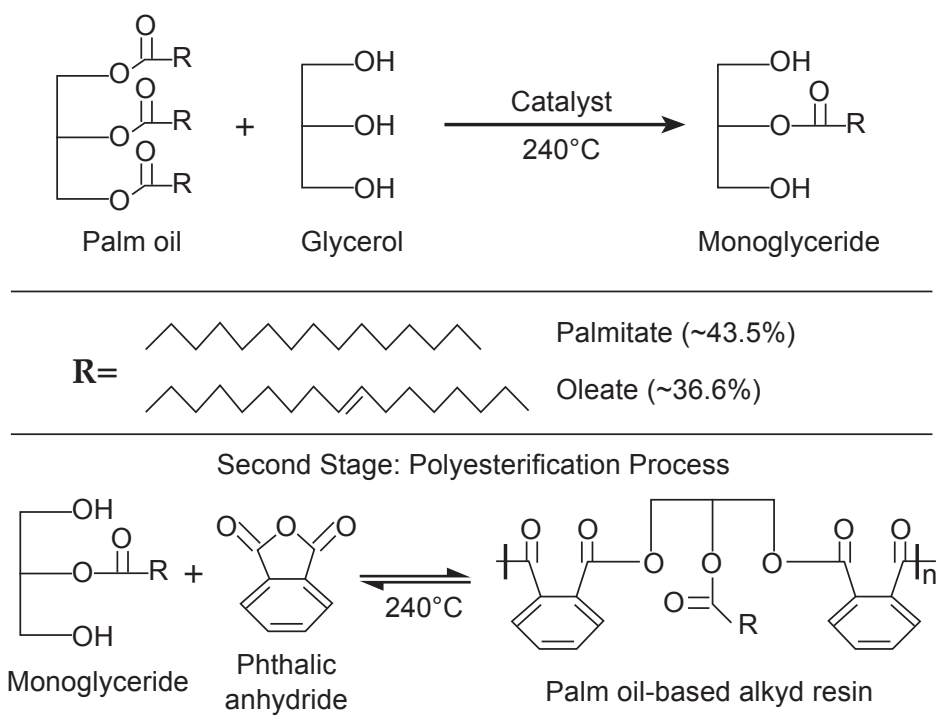

Source: Ong et al. (2015d).

Figure 2. Synthesis pathway of palm oil-based alkyd resin (alcoholysis-polyesterification process). 
where, $\mathrm{C} 0=$ acid value at the beginning and $\mathrm{Ct}=$ acid value at the time $t, t=$ reaction time.

\section{Molecular Weight $\left(\mathrm{M}_{\mathrm{w}}\right)$ Determination}

A waters gel permeation chromatography (GPC) was used to determine the molecular weight $\left(M_{w}\right)$ of the formulated alkyd resins. The testing was performed at room temperature using tetrahydrofuran as an eluent. Linear polystyrene was used for the calibration. Waters empower software was used for the data analysis.

\section{FTIR Spectroscopy}

The functional groups of palm oil and the resin (A-60) were observed by a Fourier transform infrared (FTIR) spectroscopy, model- Spectrum 100, Perkin Elmer. The scanning range was 600 to 4000 $\mathrm{cm}^{-1}$ with a resolution of $4 \mathrm{~cm}^{-1}$.

\section{Curing Process}

MEKP was used for the curing process of the prepared alkyd resins. Exactly, $10 \mathrm{~g}$ of alkyd resin was poured in a beaker. After that, $4 \mathrm{phr}$ of MEKP, $30 \mathrm{phr}$ of styrene and $2 \mathrm{phr}$ of cobalt oxalate was mixed with the resin and stirred for $10 \mathrm{~min}$ at $60^{\circ} \mathrm{C}$. After that, the resin mixture was poured to a petri dish for the curing process. The samples were observed and fingertip was used to check whether the films had solidified or not. The spot less samples were accounted as the cured film eventually.

\section{Pencil Hardness Testing}

The hardness of the formulated alkyd films was determined by a set of pencils (6B to $6 \mathrm{H}$ ) and pencil hardness tester, model- B-3084. To test the samples, different pencils were placed inside the tester at $45^{\circ}$ angle and dragged onto the surface of the films. If the pencils were able to make a scratch onto the surface of the films, they are considered as 'fail'. The negative result of this are considered as 'pass'. The methods are stated in ASTM D3363.

\section{Chemical Resistivity Test of Alkyd Film}

For chemical resistivity testing, four different types of solution were prepared. The different types chemical solution were distilled water, aqueous Hydrogen chloride (HCL) $(5 \%)$, brine solution $(5 \%)$ and $\mathrm{NaOH}(5 \%)$. The prepared alkyd films which weighted nearly $0.5 \mathrm{~g}$, were immersed into the solutions at ambient temperature for one day only. After that, the weights of the samples were measured. A qualitative performance according to their appearance after the immersion period was noted as the observations from the testing.

\section{RESULTS AND DISCUSSION}

\section{Preparation of Alkyd Resin}

The MG, DG and TG concentration at different time intervals were determined by HPLC and the data are presented in Figure 3. It can be seen that, TG concentration dropped sharply as the reaction progressed and reached after $30 \mathrm{~min}$ of reaction. The MG and DG concentrations had increased and reached plateau after 40 and $30 \mathrm{~min}$, respectively. In order to investigate the effect of MG:DG ratio on polyesterification reaction, alcoholysis reaction was stopped at 30, 40 and $60 \mathrm{~min}$ and proceeded to polyesterification reaction. The samples at 30, 40 and 60 min were labeled as A-30, A-40 and A-60, respectively.

The AV of the reaction products was checked frequently to trace the progress of polyesterification. The unreacted carboxylic acid functional groups were estimated for this purpose. Figure 4 presents the $\mathrm{AV}$ of the reaction products at different time intervals to show the effects of MG:DG ration on polyesterification. The primary and secondary hydroxyl group show different reactivity (Ong et al., 2016b). Therefore, the AV of the reaction products changed as the reaction progressed. It was found that the $\mathrm{AVs}$ decreased as the reaction proceeded. The rate of decrease of AV was faster in the early period of the polyesterification compare to the later period. This is because of the superior reactivity of the primary hydroxyl group. The similar trends were also reported elsewhere. The viscosity of the reaction products increased due to the possible formation of a 3D crosslinked network structure at the advanced stages of the polyesterification.

The effects of the ratio of MG:DG are seen in the early period of the reaction in Figure 4. The threepoint method was adopted to evaluate the initial rate of the reactions. The results are presented in Table 3. The MG:DG ratio for A-30, A- 40 and A- 60 was 1.91, 2.66 and 2.65, respectively. The initial reaction rates of A-30, A-40 and A-60 was 6.8169, 12.7860 and $14.3379 \mathrm{mg} \mathrm{KOH} \mathrm{g}^{-1} \mathrm{~s}^{-1}$, respectively. It can be seen that, the initial reaction rates for high MG:DG ratio (A-40 and A-60) were significantly higher than the lower MG:DG ratio (A-30). In addition, the initial reaction rate of $\mathrm{A}-40$ was double than A-30. Comparing all ratios, A-40 and A-60 achieved the equilibrium after $1 \mathrm{hr}$. The DP of the reactions was determined using Equations 1 and 2.

The influences of MG:DG ratio on the DP are also illustrated in Figure 4. As expected, the DP had increased with the reaction propagation. Two separate stages were identified in the DP analysis. The first straight nature of the curve indicates the formation of bonds between PA and primary hydroxyl groups. The rate of the reaction was 


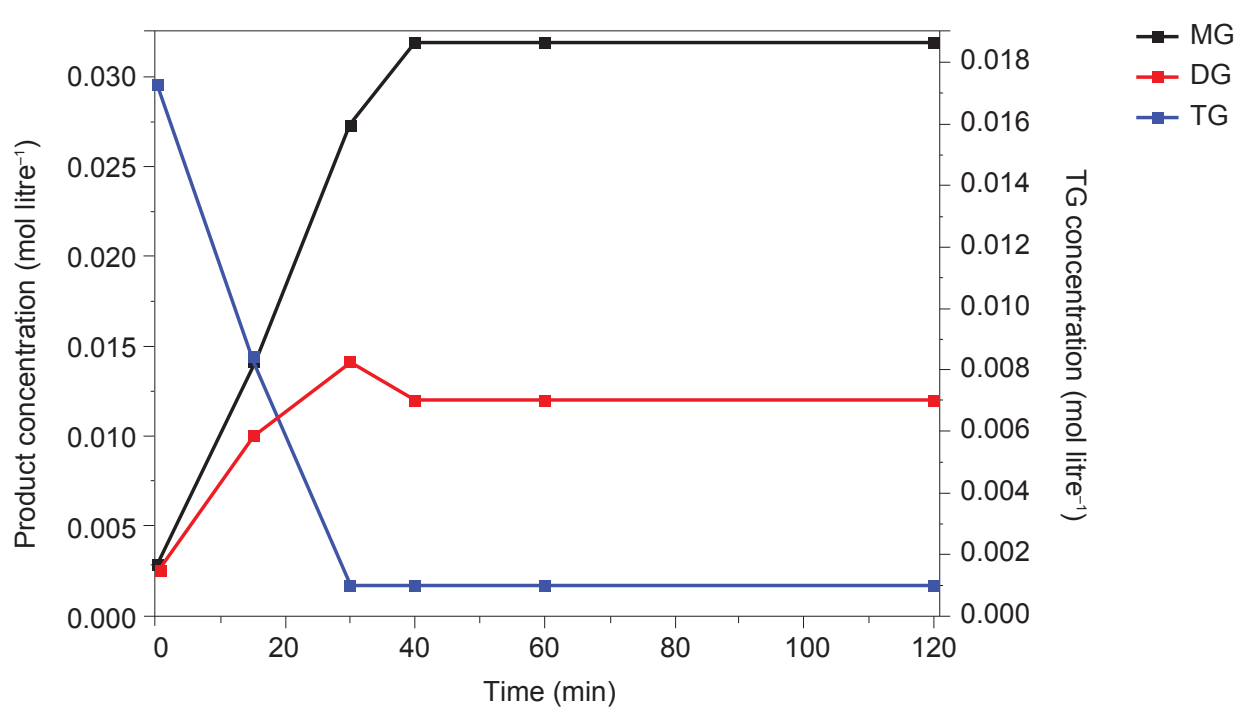

Figure 3. Plot of concentration of monoglyceride (MG), diglyceride (DG) and triglyceride (TG) vs. time over $\mathrm{CuO}-\mathrm{NaOH}$ catalyst system.

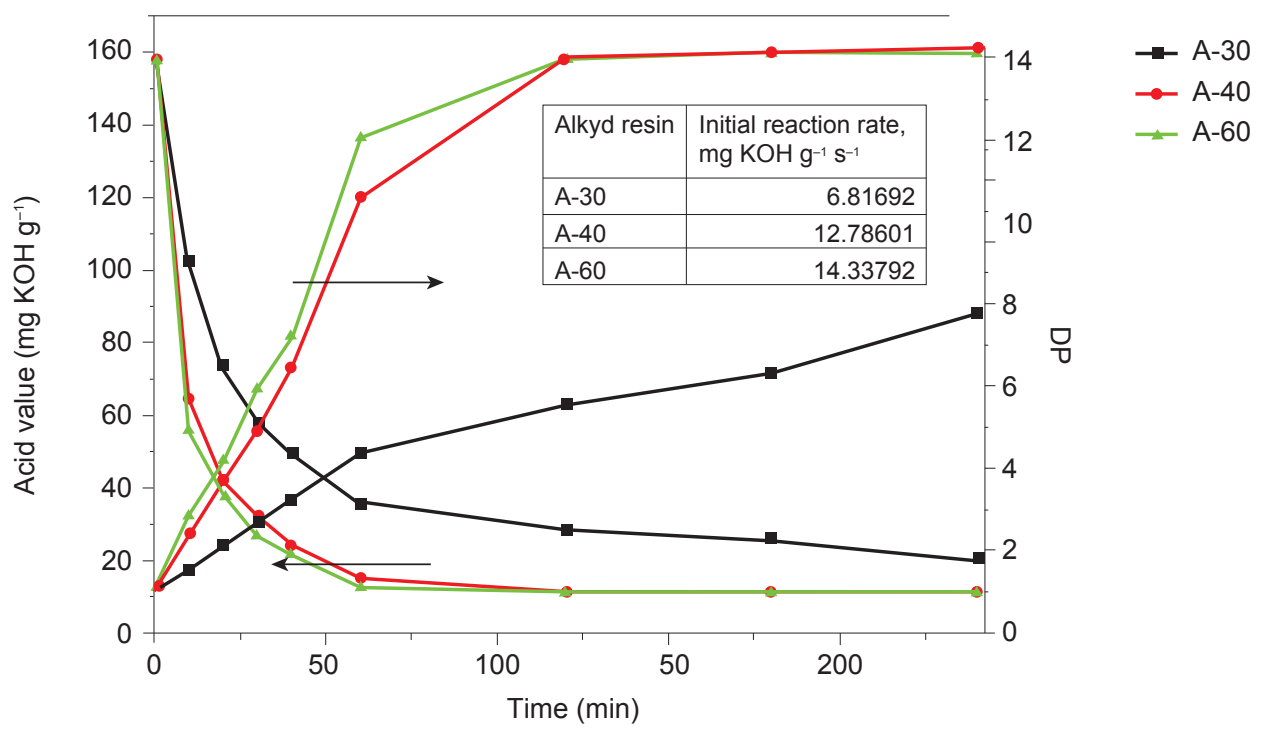

Figure 4. Plot of acid value and degree of polymerisation (DP) against reaction time for different monoglyceride/ diglyceride ratio influenced polyesterification process $(\bullet A-30, \bullet A-40$, and $\mathbf{\Delta} A-60)$. Table of initial reaction rates is shown inside the figure.

substantially influenced by the MG:DG ratio. It can be inferred that high MG:DG ratio is responsible for high rate of reaction. On the other hand, the second stage indicates the reaction between the PA and secondary group of MG. In this stage, a high DP value was observed up to $2 \mathrm{hr}$ of reaction time for the case of A-60. Therefore, A-60 was chosen as the optimal ratio.

\section{Molecular Weight $\left(\mathrm{M}_{\mathrm{w}}\right)$ Characterisation}

Table 1 presents the weight molecular weight $\left(\mathrm{M}_{\mathrm{w}}\right)$ of the alkyd resin at various reaction times. It can be seen that as the reaction preceded the increased $M_{w}$ of the synthesised alkyd resin (A-30 and A-60). Initially, at the start of the polymerisation reaction, the rate of the polymerisation was low.
Therefore, the $M_{w}$ of the formulated resin was also low and as the rate increased, the $\mathrm{M}_{\mathrm{w}}$ of the polymer also increased. Previous researchers whom studied with vegetable oil-based alkyds recorded similar observations. It is noticed that, the $\mathrm{M}_{\mathrm{w}}$ of A-60 was higher than A-30 at all time intervals. This phenomenon might be due to that the MG/DG ratio of A-60 (2.65) was higher than A-30 (1.91). It is well known that, MG and DG in the mixture could readily react with phthalic anhydride during polyesterification reaction and DG acted as a chain stopper. The chain length of the alkyd was significantly influenced by the ratio of MG to DG in the mixture, which directly affected the $M_{w}$ of alkyd. Therefore, the A-60 sample with higher MG:DG ratio possessed higher $M_{w}$ than A-30 sample. 
TABLE 1. MOLECULAR WEIGHT ( $\left.M_{w}\right)$ OF ALKYD RESIN, A-30 AND A-60 AT VARIOUS INTERVALS TIME

\begin{tabular}{ccc}
\hline \multirow{2}{*}{ Time (min) } & \multicolumn{2}{c}{$\mathbf{M}_{\mathrm{w}}\left(\mathrm{g} \mathrm{mol}^{-\mathbf{1}}\right)$} \\
\cline { 2 - 3 } & $\mathbf{A - 3 0}$ & $\mathbf{A - 6 0}$ \\
\hline 0 & 740 & 740 \\
60 & 1202 & 1406 \\
120 & 1373 & 1655 \\
180 & 1988 & 2108 \\
\hline
\end{tabular}

\section{FTIR Analysis}

Figure 5 shows the FTIR spectra of A-60 and palm oil. The absorbance peak at $3650-3350 \mathrm{~cm}^{-1}$ (Ong et al., 2015c; Khan et al., 2016; Ong et al., 2016c) confirmed the presence of hydroxyl group. The strong absorbance peak in the range of 3050-2780 $\mathrm{cm}^{-1}$ detected the $\mathrm{C}-\mathrm{H}$ stretching. The stretching vibration of $\mathrm{C}-\mathrm{O}-\mathrm{C}$ and $\mathrm{C}=\mathrm{O}$ functional group in ester of TG was confirmed by the peak at 1280-1100 $\mathrm{cm}^{-1}$ and 1750-1700 $\mathrm{cm}^{-1}$, respectively (Assanvo et al., 2015). The absorption of $\mathrm{C}-\mathrm{H}$ bending was at $1465-1445 \mathrm{~cm}^{-1}$. The peak at $735 \mathrm{~cm}^{-1}$, detected the unsaturated condition of the fatty acids. A new characteristic peak for the ester functional group appeared and confirmed by the peak at 1255$1225 \mathrm{~cm}^{-1}$. The peak at $750 \mathrm{~cm}^{-1}$, confirmed the $\mathrm{C}-\mathrm{H}$ bending in the aromatic group. The peak can confirm the incorporation of phthalate group and the polyesterification.

\section{Pencil Hardness Test}

The pencil hardness testing was performed for the measurement of the hardness of the films. The results are presented in Table 2. The analysis showed
A-60 film has better hardness than A-30 film, as A-60 film passed pencil category B. This might be due to higher crosslinking in A-60 film compared to A-30 film.

TABLE 2. PENCIL HARDNESS OF A-30 AND A-60 CURED FILM

\begin{tabular}{ccc}
\hline Pencil type & A-30 film & A-60 film \\
\hline 2B & Pass & Pass \\
B & Fail & Pass \\
HB & Fail & Fail \\
\hline
\end{tabular}

\section{Chemical Resistivity}

Table 3 presents the performance of chemical resistivity of A-30 and A-60. Results showed that both films were highly resistant to the chemicals (hydrochloric acid and aqueous sodium chloride) and distilled water, but the results were different for the case of $\mathrm{NaOH}$. The resistivity was different for different samples. A-30 film showed poor resistivity to alkali solution, while A-60 film showed comparatively fair resistivity to the same solution. This is because of the hydrolysis process of the ester functional groups present in the alkyds (Essien et al., 2015).

TABLE 3. CHEMICAL RESISTIVITY OF A-30 AND A-60 FILMS

\begin{tabular}{lcc}
\hline Solution & A-30 film & A-60 film \\
\hline Distilled water & Excellent & Excellent \\
$5 \%$ of $\mathrm{HCl}$ (aq.) & Excellent & Excellent \\
$5 \% \mathrm{NaOH}$ (aq.) & Poor & Fair \\
$5 \% \mathrm{NaCl}$ (aq.) & Excellent & Excellent \\
\hline
\end{tabular}

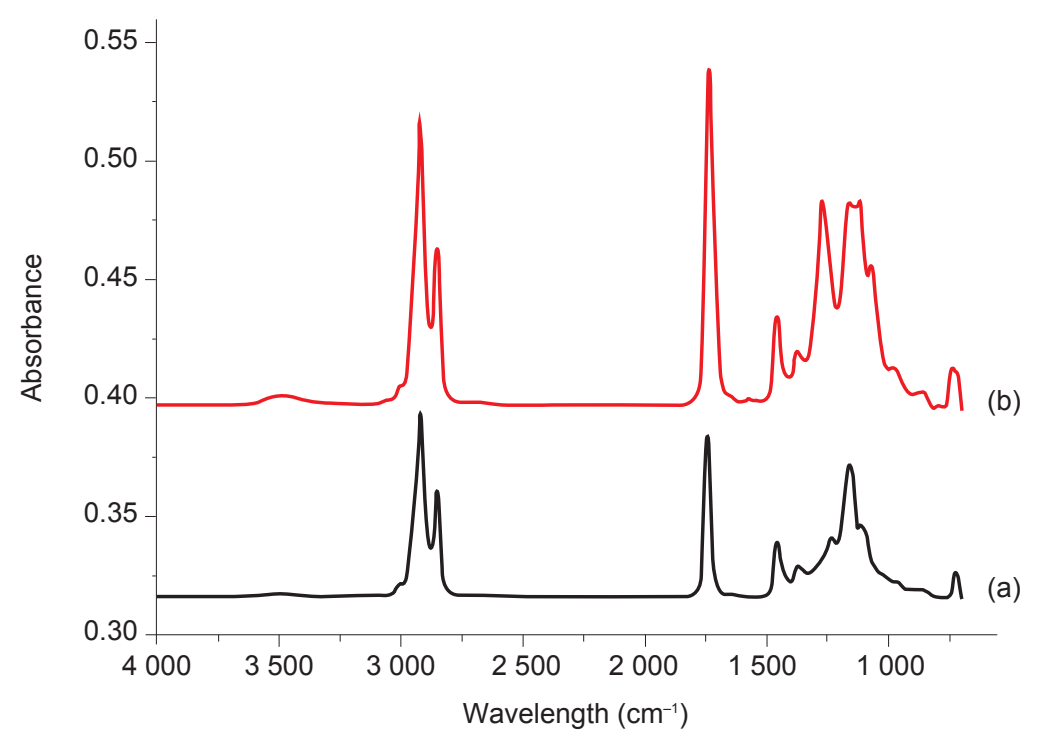

Figure 5. Infrared spectra of (a) palm oil, and (b) A-60 alkyd. 


\section{CONCLUSION}

The scope of this work is to find the effects of the variation of MG/DG ratio on the properties of palm oil-based polyalkyds. The synthesis process includes alcoholysis followed by polyesterfication. Different properties of polyalkyds like $\mathrm{M}_{\mathrm{w}}$, DP, structural, hardness and chemical resistivity were evaluated. The FTIR analysis confirmed the presence of ester functional groups in the prepared alkyd resins. It was found that, the reaction time had shortened due to the higher initial rate at higher MG:DG ratio. The initial rate for A-60 (MG:DG=2.65) was two times higher than the A-30 (MG:DG=1.91). In addition, higher MG/DG ratio in the polyesterification reactions produced the alkyd resin with higher $\mathrm{M}_{\mathrm{w}}$. Moreover, A-60 film possessed better pencil hardness and chemical resistivity than A-30 film. It can be concluded that a higher ratio of MG:DG resulted in a higher $\mathrm{M}_{\mathrm{w}}$ polyalkyds, which can be suitable for coating application.

\section{ACKNOWLEDGEMENT}

This work was financially supported by research grant from Universiti Malaysia Pahang, Malaysia (Project No: GRS 130350) and GSAS scholarship from Malaysian Palm Oil Board for which the authors are very grateful.

\section{REFERENCES}

Assanvo, E F; Gogoi, P; Dolui, S K and Baruah, S D (2015). Synthesis, characterization and performance characteristics of alkyd resins based on Ricinodendron Heudelotii oil and their blending with epoxy resins. Ind. Crops Prod., 65: 293-302.

Beg, M D H; Islam, M R; Ahmed, B; Yunus, R M and Abdullah, A (2019). Optimization of reaction parameters of esterification on the synthesis of palm oil-based alkyds using response surface methods. 2019 IOP Conf. Ser.: Mater. Sci. Eng., 634: 012038.

Dolui, S K (2015). Blends of epoxidized alkyd resins based on jatropha oil and the epoxidized oil cured with aqueous citric acid solution - A green technology approach. ACS Sustain. Chem. Engi., 3: 261-268.

Essien, E E; Umoren, S A and Effiong, E E (2015). Synthesis and characterization of Luffa Cylindrica fatty acids-based alkyd resins. Res. Chem. Intermed. p. 1-13.
Khan, M M R; Rahman, M W; Ong, H R; Ismail, A $B$ and Cheng, C K (2016). Tea dust as a potential low-cost adsorbent for the removal of crystal violet from aqueous solution. Desalination Water Treat., 57(31): 14728-14738.

Meneghetti, M R and Meneghetti, S M P (2015). Sn (iv)-based organometallics as catalysts for the production of fatty acid alkyl esters. Catal. Sci. Techno., 5: 765-771.

Mutar, M A and Hassan, N M A (2017), Synthesis and characterization of new alkyd resins (short, medium and long) based on sunflower oil and linoleic acid as binder for paints. Int. J. Chem. Petrochemical Technol., 7(1): 1-16.

Ong, H R; Khan, M M R; Ramli, R; Hong, C S and Yunus, R M (2018). Influence of $\mathrm{CuO}$ nanoparticle on palm oil based alkyd resin preparation and its antimicrobial activity. IOP Conf. Series: Mater. Sci. Eng., 324: 012027 DOI:10.1088/1757899X/324/1/012027.

Ong, H R; Khan, M M R; Ramli, R; Du, Y; Xi, S and Yunus, R M (2015a). Facile synthesis of copper nanoparticles in glycerol at room temperature: Formation mechanism. RSC Adv., 5(31): 2454424549.

Ong, H R; Khan, M M R; Ramli, R and Yunus, R M (2015b). Effect of $\mathrm{CuO}$ nanoparticle on mechanical and thermal properties of palm oil based alkyd/epoxy resin blend. Procedia Chem., 16: 623-631.

Ong, H R; Khan, M R; Yousuf, A; Jeyaratnam, N and Prasad, D M (2015c). Effect of waste rubber powder as filler for plywood application. Polish J. Chemical Technol., 17(1): 41-47.

Ong, H R; Khan, M M R; Ramli, R; Rahman, M W and Yunus, R M (2015d). Tailoring base catalyzed synthesis of palm oil-based alkyd resin through CuO nanoparticles. RSC Adv., 5(116): 95894-95902. DOI:10.1039/c5ra19575f.

Ong, H R; Khan, M M R; Ramli, R; Yunus, R M and Rahman, M W (2016a). Glycerolysis of palm oil using copper oxide nanoparticles combined with homogeneous base catalyst. New J. Chem., 40(10). DOI:10.1039/C6NJ01461E.

Ong, H R; Ramli, R; Khan, M M R and Yunus, R $\mathrm{M}$ (2016b). The influence of $\mathrm{CuO}$ nanoparticle on non-edible rubber seed oil based alkyd resin preparation and its antimicrobial activity. Prog. Org. Coat., 101: 245-252. 
Ong, H R; Hegde, G; Chigrinov, V and Khan, M M R (2016c). Sulfuric disazo dye stabilized copper nanoparticle composite mixture: Synthesis and characterization. RSC Adv., 6: 15094-15100.
Rarokar, N R; Menghani, S; Kerzare, D and Pramod Bhujangrao Khedekar, P B (2017). Progress in synthesis of monoglycerides for use in food and pharmaceuticals. J. Food Pharmaceutical Sci., 5: 13-19.

\section{JOURNAL OF} OIL PALM RESEARCH Micnosite

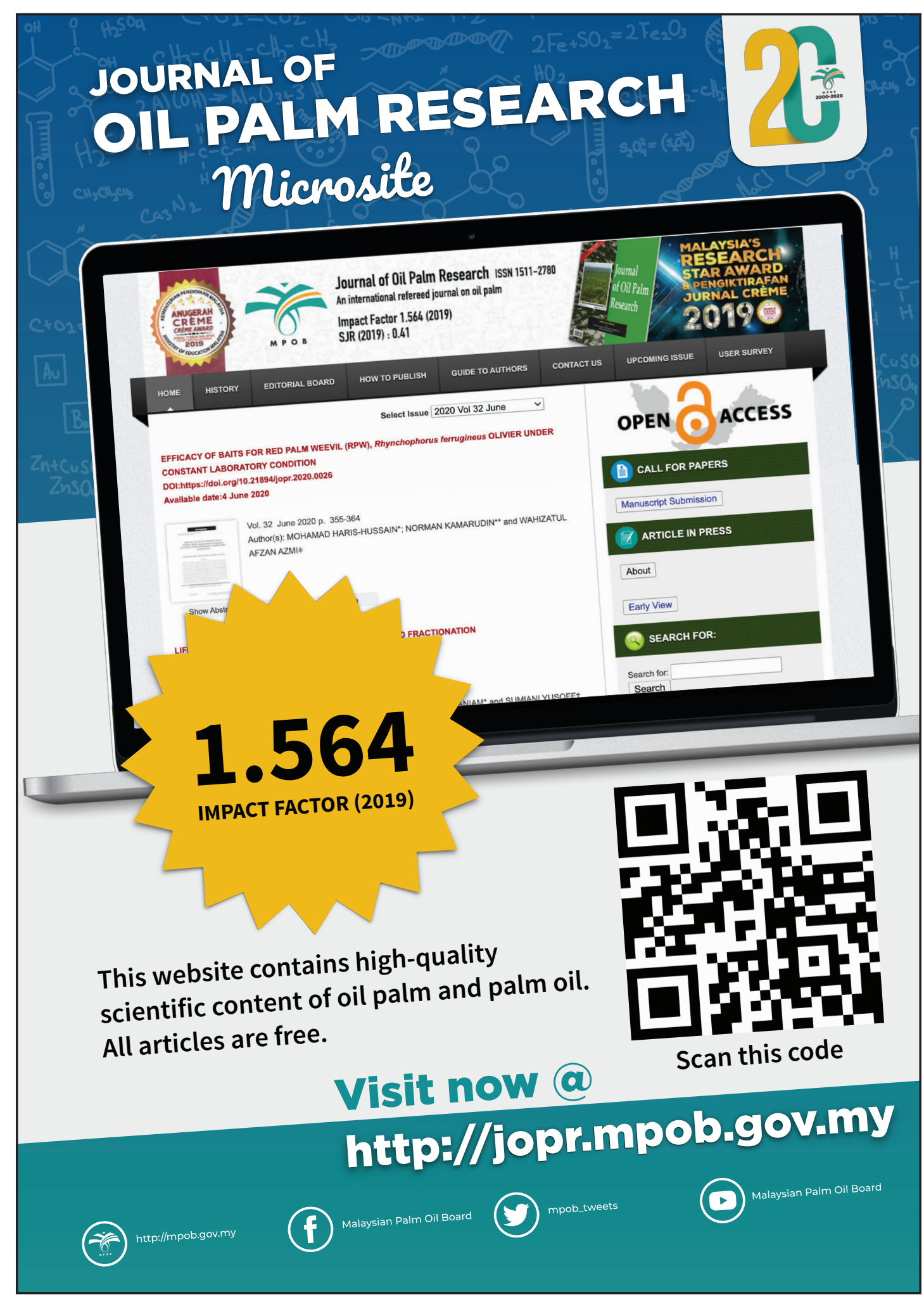

